\title{
Osteosynthesen am proximalen Femur
}

\author{
K. Weise, E. Schwab
}

\section{Zusammenfassung}

Die Fortentwicklung geeigneter Osteosynthesetechniken bei Frakturen im Trochanterbereich hat zu einer spürbaren Verbesserung der Ergebnisse sowie zu einer deutlichen Reduktion der Komplikationsraten beigetragen. Dennoch sind auch diese nicht ganz frei von Problemen. Gerade beim alten Patienten mit herabgesetzter Knochenqualität, eingeschränkter Kooperationsfähigkeit sowie dem Anspruch auf eine voll belastbare Extremität bieten intramedulläre Stabilisierungsverfahren erhebliche Vorteile. Für die Auswahl der im Einzelfall geeigneten Versorgungstechnik muss das Ausmaß der durch den Bruchtyp hervorgerufenen Instabilität Berücksichtigung finden. Trochantere Frakturen reichen von der stabilen nicht oder wenig dislozierten Form bis zu den hochgradig instabilen intertrochanteren Bruchtypen. Subtrochantere Frakturen sind vielfach mit solchen im intertrochanteren Bereich kombiniert. Eine solche Bruchkonstellation stellt aufgrund der hochgradigen Instabilität ein besonderes Problem dar. Frakturen am proximalen Femur können mit extramedullär gelegenen Implantaten, welche in starrer (95-Grad-Condylenplatte, 130-Grad-Winkelplatte) oder in dynamischer Form vorliegen (DHS ohne/mit Trochanterstabilisierungsplatte) oder als intramedulläre Versorgungstechnik (proximaler Femurnagel - PFN, Gamma-Nagel, u.a.) Verwendung finden. Je instabiler eine Fraktur, desto eher kommen intramedulläre dynamische Implantate zur Anwendung.

\section{Einleitung}

Hüftgelenksnahe Frakturen des Oberschenkels gehören gleichermaßen zu den häufigen wie aufgrund der biomechanischen Bedingungen und der betroffenen Altersgruppe anspruchsvollen knöchernen Verletzungen. Am coxalen Femurende unterscheidet man Frakturen des Hüftkopfes von solchen des Schenkelhalses und Frakturen im Trochanterbereich. Die Arbeitsgruppe „Osteosynthesen am proximalen Femur" der Deutschen Sektion der Internationalen Arbeitsgemeinschaft für Osteosynthesefragen (AO) hat sich mit Verletzungen der letzteren Region befasst.

OP-JOURNAL 2000; 16: 252-258

(c) Georg Thieme Verlag Stuttgart · New York
Die besonderen Umstände bei Frakturen des alten Menschen mit seinen Vorerkrankungen, seinem eingeschränkten Allgemeinzustand, der minderen Knochenqualität, herabgesetzter koordinativer Fähigkeiten und nur bedingt vorhandener Kooperationsmöglichkeiten bieten für jegliche Art operativer Versorgung teilweise äußerst schwierige Rahmenbedingungen. Andererseits ist bei zunehmender Lebenserwartung gerade diese Altersgruppe von einer ansteigenden Zahl knöcherner Verletzungen des proximalen Femurs betroffen. Dies wird erhellt aus einigen demographischen Daten, welche die zukünftige Entwicklung der Alterstraumatologie verdeutlichen:

Die durchschnittliche Lebenserwartung ab der Geburt beträgt in Deutschland heute rund 80 Jahre für Frauen, gut 73 Jahre für das männliche Geschlecht. Für einen heute 65-jährigen Mann ist eine mittlere „Rest-Lebenserwartung“ von 15 Jahren, für eine Frau gleichen Alters sogar eine über 18 Jahre zugrunde zu legen. In den USA können 85-jährige von einer weiteren Lebenserwartung von $61 / 2$ Jahren (weibliches Geschlecht) und gut 5 Jahren (männliches Geschlecht) ausgehen.

Insgesamt machen ältere Menschen einen unverhältnismäßig hohen Prozentsatz an Unfallverletzten aus. Während der Bevölkerungsanteil der über 65-jährigen laut statistischem Jahrbuch im Jahr 1998 etwa $17 \%$ der gesamten Bevölkerung bildete, liegt ihr Anteil an der Belegung von Krankenhausbetten bei ca. $40 \%$. In den Vereinigten Staaten beanspruchen $\mathrm{Pa}$ tienten aus dieser Altersgruppe - prozentual gesehen - bereits einen etwa doppelt so hohen Anteil am Unfallgeschehen als ihrer tatsächlichen Zahl eigentlich zukäme. Ursachen für die besonders ausgeprägte Unfallgefährdung des alten Menschen sind zum einen die allgemeine $\mathrm{Ge}-$ brechlichkeit und Hinfälligkeit, aber auch die Tatsache reduzierter koordinativer Fertigkeiten sowie akut eintretende Be-
Anforderungen an ein ideales Implantat bei trochanteren Frakturen:

- Gewährung der Belastungsstabilität

- Sichere Verankerung bei Osteoporose

- Ermöglichung der Fraktursinterung

- Vermeidung einer Implantatperforation in das Gelenk

- Schonung der Vaskularisation

- Einfache Implantationstechnik Stürmer, 1993 [8] 
gleiterkrankungen wie kardiale Zwischenfälle, ein Apoplex oder eine Kreislaufregulationsstörung. Nicht zuletzt spielt der Einfluss von Medikamenten bei der Sturzursache eine bedeutende Rolle. Der ältere Mensch ist sowohl hinsichtlich der aktiven Teilnahme im Straßenverkehr mittels Pkw oder Zweirad als auch als Fussgänger in hohem Maße gefährdet. Aus verschiedenen epidemiologischen Studien geht hervor, dass knapp 4\% der älteren Bevölkerung wegen Sturzverletzungen ärztliche Behandlung in Anspruch nehmen müssen und dass sie mehr als $50 \%$ der Unfalltoten und sogar $2 / 3$ der stationär $\mathrm{zu}$ behandelnden Unfallverletzten ausmachen. Die Unfallgefährdung älterer Mitbürger im Straßenverkehr wird mit derjenigen von Kindern verglichen. Die durchschnittlichen Behandlungskosten des älteren unfallverletzten Patienten liegen infolge der höheren Komplikationsrate, der längeren Liegedauer auf der Intensivstation sowie der mannigfachen Probleme in der Rehabilitation um ein Mehrfaches höher als beim jüngeren Verletzten.

Derlei Zahlen verdeutlichen, dass der betagte Mensch als Patient gerade auch in der Unfallchirurgie eine zunehmende Bedeutung erlangen wird. Darüber hinaus hat dieser Umstand gleichermaßen Konsequenzen hinsichtlich der Probleme und Schwierigkeiten bei der operativen Versorgung einschließlich der Begleit- und Nachbehandlung wie auch bezüglich der wirtschaftlichen Auswirkungen mit einer enormen Kostenbelastung des Gesundheitswesens. Dies ist auch Ursache für prophylaktische Überlegungen im Hinblick auf die sog. Sturzfraktur des alten Menschen, welche in Skandinavien bei gefährdeten Patienten in höherem Lebensalter in einer Art gepolsterter Hose besteht, mittels derer eine signifikante Senkung von Frakturen am coxalen Femurende zu verzeichnen ist. Die geriatrische Forschung beschäftigt sich mit weiteren Möglichkeiten der Prävention, ebenso wie mit Programmen der Rehabilitation, welche den Erhalt der psychosozialen Kompetenz greiser Patienten zum Ziel haben.

Frakturen am coxalen Femurende können mit Vorteil nach der AO-Klassifikation eingeteilt werden, welche eindeutige Aussagen zum Instabilitätsgrad der Verletzung zulässt. Die Frakturen der Trochanterregion untergliedert man in einfache und multifragmentäre, wobei letztere infolge der Beteiligung beider Trochanteren besonders instabil sind. Sie

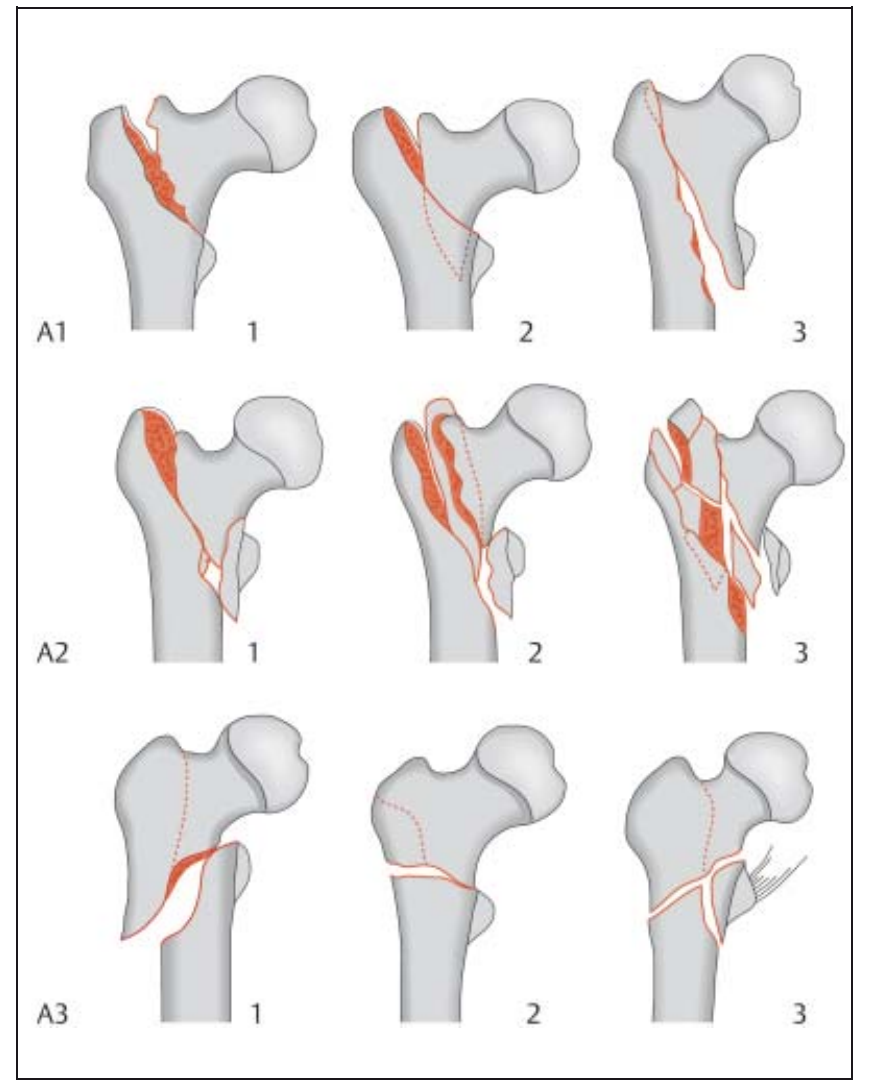

Abb. 1 Ao-Klassifikation von Frakturen der Trochanterregion.

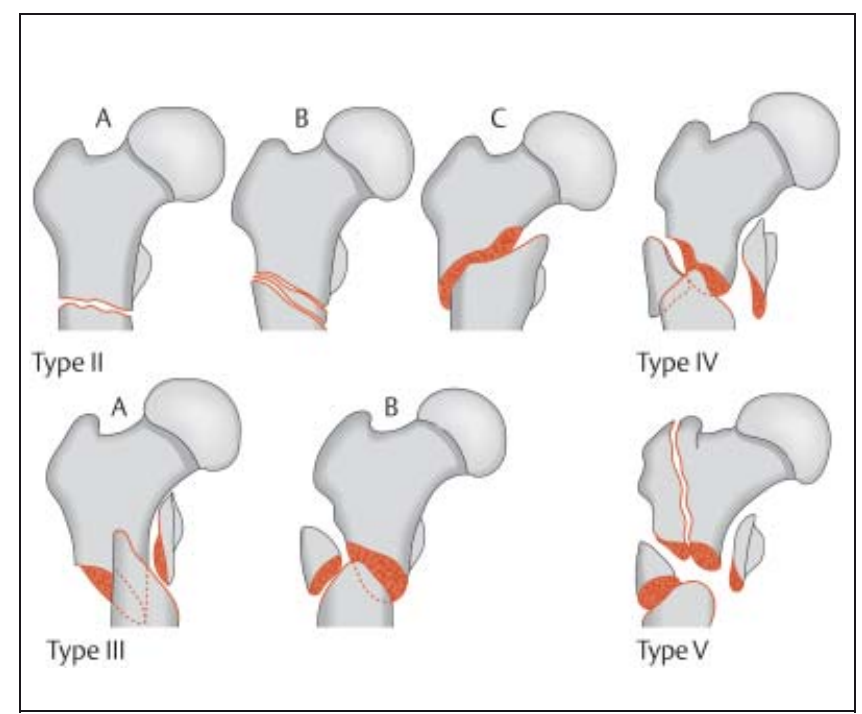

Abb. 2 Klassifikation subtrochanterer Frakturen nach SENNHEISER.

müssen demzufolge mit einem volle Belastbarkeit garantierenden Implantat versorgt werden. Die 3. Gruppe der trochanteren Frakturen sind die sog. intertrochanteren Verletzungstypen, welche auch als „reversed fractures“ bezeichnet werden. Dies bedeutet, dass die Bruchlinie im Unterschied zu den beiden vorgenannten Gruppen gegenläufig („verdreht“) verläuft, was gleichfalls zu ausgeprägter Instabilität führt (Abb.1).
Die eigentlichen subtrochanteren Brüche weisen aufgrund ihrer engen Lagebeziehung zur Trochanterregion in einem hohen Prozentsatz Kombinationen mit intertrochanteren Frakturen auf, welche Kombination im Hinblick auf die Instabilität besonders gravierend ist. Subtrochantere Frakturen werden nach Sennheiser in 5 Gruppen unterteilt, wobei die intertrochantere Region in diese Klassifikation mit einbezogen ist (Abb.2). 
Die biomechanischen Bedingungen am proximalen Femur sind im Falle einer Fraktur deswegen so ungünstig, weil es sich an diesem anatomischen Ort um eine exzentrische Belastung handelt, indem die Tragachse medial des Knochens verläuft. Der Schenkelhals wirkt dabei wie der Ausleger eines Krans; dies bedeutet, dass auf der Lateralseite eine Zugspannung entsteht, welche von der dort verlaufenden Muskulatur aufgefangen werden muss, wohingegen die Medialseite unter Druckspannung gelangt. Dieser Umstand legt nahe, dass im Falle einer Fraktur das zur Stabilisierung verwendete Implantat eigentlich auf die Innenseite des körpernahen Femurknochens gelegt werden müsste, um aufgrund der Nähe zur Belastungsachse als Abstützung geeignet zu sein. Dies ist aus Gründen der Weichteile und des operativen Zugangsweges nicht praktikabel bzw. wäre nur unter größeren Schwierigkeiten umzusetzen. Die Bestrebung muss dennoch sein, bei höherem Instabilitätsgrad das Implantat möglichst nahe an diese Belastungsachse zu verbringen. Diese Anforderung ist mit einem im Markraum des Femurs gelegenen sog. intramedullären Implantat am ehesten zu verwirklichen.

Folgerichtig unterscheidet man bei der osteosynthetischen Versorgung trochanterer Frakturen intra- von extramedullär gelegenen Implantaten. Letztere liegen mit einer Platte an der Außenseite des proximalen Femurs und sind mit Schrauben oder einer festen Klinge ausgestattet, welche ihrerseits im Schenkelhals verankert werden. Es handelt sich also um statisch wirkende Implantate aus einem Werkstück (Platte mit Klinge) oder um die Verwirklichung eines dynamischen Prinzips, in dem die extramedullär gelegene Platte mit einem beweglichen $\mathrm{Zu}$ satzimplantat (Schraube oder Doppelklinge) in Verbindung steht. Das dynamische Stabilitätsprinzip bietet den Vorteil, dass im Falle eines instabilen Bruches mit einer Trümmerzone unter Belastung ein sog. „Teleskoping“ stattfinden kann, welches ein Zusammenrutschen der Fraktur und damit ein höheres Maß an Stabilität wie auch einen besseren Fragmentkontakt und damit günstigere Bedingungen für die knöcherne Heilung ermöglicht.

Man unterscheidet neben intra- und extramedullär gelegenen Implantaten demnach solche, welche dynamisch wirksam sind von solchen rein statischer Art. Je instabiler trochantere Frakturen, umso eher sollte ein intramedullär verankertes Implantat und ein solches mit dynami- schem Verankerungsprinzip ausgewählt werden. Nachstehende häufig verwendete Implantate sind auf dem Markt:

\section{Extramedullär}

- dynamische Hüftschraube (DHS)

- dynamische Hüftschraube mit (Trochanterstabilisierungsplatte DHS + TSP)

- dynamische Condylenschraube (DCS)

130-Grad-Winkelplatte (statisch)

95-Grad-Condylenplatte (statisch)

Intramedullär

- proximaler Femurnagel (PFN)

- Gamma-Nagel

- Classic-Nail

- Gleitlaschennagel

- Targon-Nagel

Aufgrund der Rotationsinstabilität von A2.2-, A2.3- und A3-Frakturen hat es sich als vorteilhaft erwiesen, im Schenkelhals neben der eigentlichen Schenkelhalsschraube eine zusätzliche sog. Antirotationsschraube einzubringen, welche eine Verdrehung des Kopf-Hals-Fragmentes gegen das Schaftfragment im Verlauf des Teleskopings vermeidet. Daraus kann abgeleitet werden, dass bei zunehmendem Instabilitätsgrad speziell solche Implantate einzusetzen sind, welche diese Voraussetzung bieten. Im klinischen Alltag haben sich bei instabilen Bruchformen der intramedullär gelegene proximale Femurnagel (PFN), alternativ der Gamma-Nagel, Classic-Nail, Gleitlaschennagel und andere als besonders wirksam erwiesen. Nach eigenen Erfahrungen bietet insbesondere ersterer im Vergleich zu anderen Implantaten einige Vorteile.

Entsprechend der „Anforderungen an ein ideales Implantat“" (Stürmer [8]), zur Versorgung der Frakturen des proximalen Femurs beim alten Menschen kann man demnach für die stabileren Formen die DHS und mit zunehmender Instabilität besonders den PFN empfehlen. Für in die subtrochantere Region verlaufende Frakturen steht letzterer in einer langen Ausführung zur Verfügung. Ziel jeglicher Osteosynthese muss die volle Belastbarkeit sein, wobei der Eingriff die multimorbiden Patienten nach Möglichkeit nur wenig belasten, d.h. in einer minimal-invasiven Operationstechnik ausgeführt werden sollte. Verschiedene Untersuchungen zur Wertigkeit einzelner Verfahren bestätigen, dass bei höherer Instabilität mit Vorteil intramedullär gelegene dynamische Implantate zur Anwendung gelangen können.

\section{Erfahrungen mit dem proximalen Femurnagel (PFN) an der Berufsgenossenschaftlichen Unfallklinik Tübingen}

Vom 1.4.1996-31.12.1999 wurden 263 PFN implantiert. Es handelte sich um 186 Frauen (Alter $\varnothing 82,3$ Jahre) und 77 Männer (Alter $\varnothing$ 65,9 Jahre). Die Altersverteilung zeigt eine starke Betonung des Frauenanteils über 80 Jahren und eine gleichmäßigere Verteilung der Männer mit Verschiebung zum jüngeren Lebensalter. In 251 Fällen erfolgte die PFN-Implantation bei frischen Frakturen, in 4 Fällen zur Reosteosynthese nach primärer DHS- bzw. $95^{\circ}$-Kondylenplatte und in 8 Fällen zur Versorgung einer pathologischen subtrochanteren Fraktur bei metastasierendem Primärtumor (vorwiegend Mammakarzinom).

Neben instabilen Frakturen der AO-Klassifikation 31 A2 $(\mathrm{n}=146)$ (Abb. 3) undA3 $(n=64)($ Abb. 4) wurden auch einige A1-Frakturen $(n=27)$ mit dem PFN versorgt, um Erfahrungen mit dem intramedullären Kraftträger bei diesen stabilen Frakturen im Vergleich zur DHS zu sammeln. Nach Einführung des langen PFN konnten 16 Frakturen mit diesem Implantat stabilisiert werden, von denen 12 rein subtrochanter $(32 \mathrm{~A}-\mathrm{C})$ sowie 4 per- und subtrochanter (31 A 3 ) verliefen.

Ein Patient erlitt eine $3^{\circ}$ offene Defektfraktur der Trochanterregion, alle anderen Frakturen waren geschlossen ohne oder mit nur geringem Weichteiltrauma (G $0-G 1$ ), bei 15 Frakturen fand sich ein höhergradiges Weichteiltrauma (G 2). Es wurde immer eine Primäroperation angestrebt, teilweise verlangte jedoch die Multimorbidität der Patienten eine intensivmedizinische präoperative Vorbereitung. Immerhin konnten $82 \% \mathrm{~Pa}-$ tienten innerhalb der ersten 24 Std. nach dem Unfallereignis operiert werden, weitere $11 \%$ innerhalb des nächsten Tages.

Die Operation wurde bei 80\% der Patienten auf dem Extensionstisch durchgeführt, bei den restlichen auf dem Normaltisch. Die Operationszeit betrug durchschnittlich 65 min bei 23 verschiedenen Operateuren. Es bestand eine leichte Tendenz zur kürzeren OP-Zeit mit zunehmender Erfahrung der einzelnen Operateure entsprechend einer Lernkurve. Die durchschnittliche Bestrahlungszeit mit Bildverstärker betrug 3,45 min ebenfalls mit Tendenz zur geringeren BV-Zeit mit zunehmender Erfahrung. 

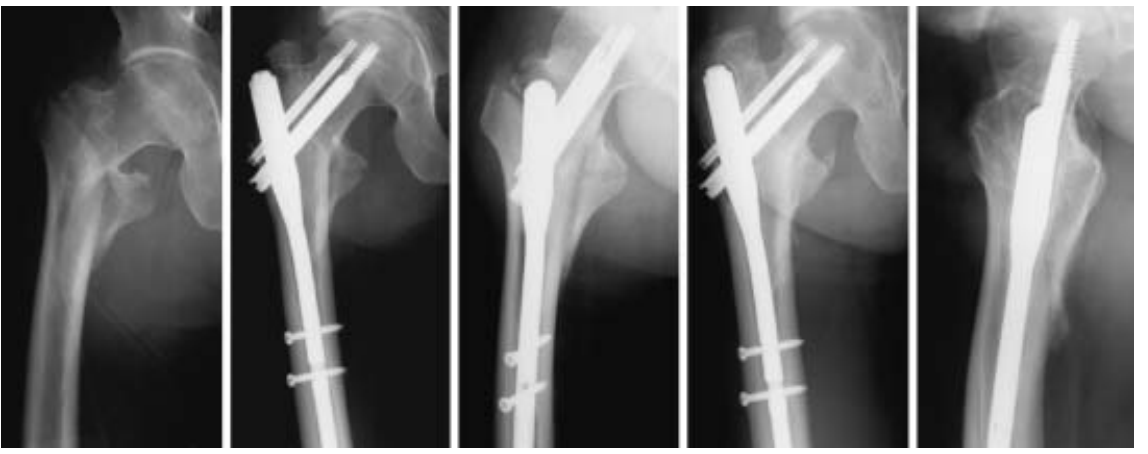

Abb. 3 Beispiel A 2 Fraktur (m, 34 J).
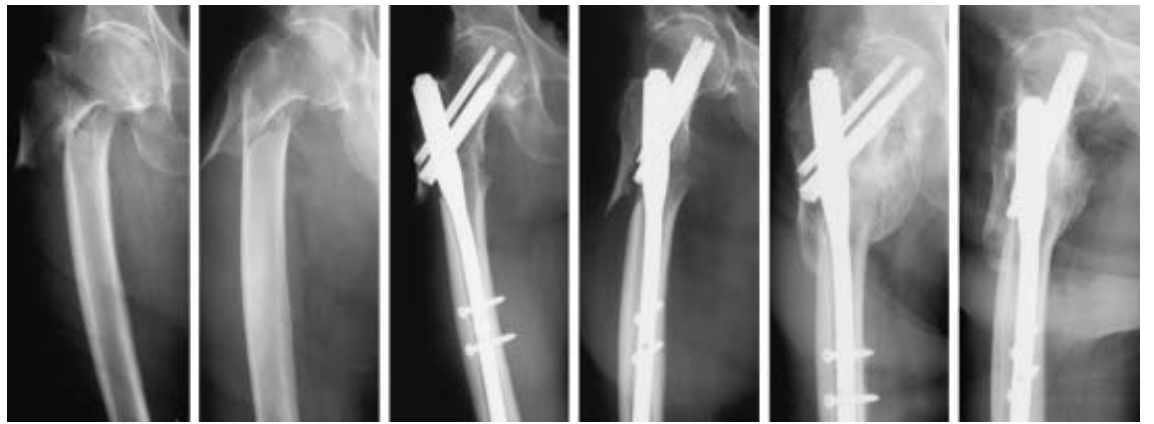

Abb. 4 Beispiel A 3 Fraktur (w, 83 J).

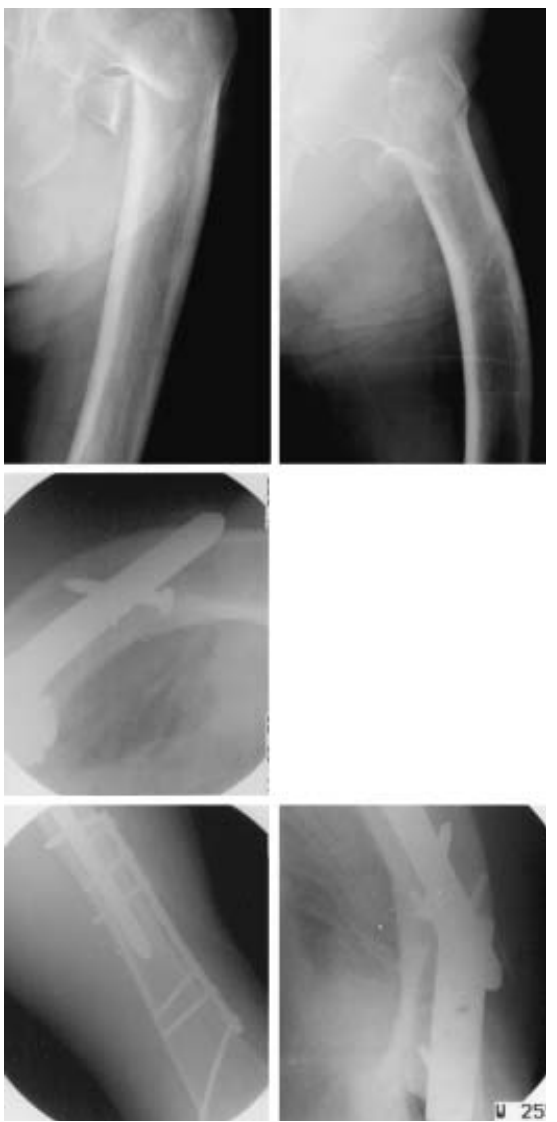

Abb. 5 Komplikation intraop. Schaftperforation, zusätzliche Plattenosteosynthese.
Postoperativ war in allen Fällen eine sofortige Gelenkmobilisation möglich, $80 \%$ der Pat. konnten innerhalb einer Woche mit Gehhilfen unter Belastung je nach Schmerzsymptomatik aufstehen, $10 \%$ benötigten aufgrund ihrer Allgemeinsituation länger als eine Woche, $10 \%$ blieben bettlägerig entsprechend ihrem präoperativen Zustand. Das Aufstehen war nie von der Osteosynthese bestimmt, bei mehr als $90 \%$ Patienten war aufgrund der erzielten Frakturstabilität die sofortige Vollbelastung erlaubt.

Intraoperativ traten keine wesentlichen technischen Schwierigkeiten auf, in 2 Fällen konnte keine zweite Schaftverriegelung durchgeführt werden, da der Zielbügel aufgrund der Antekurvation des Knochens nicht mehr mit dem Nagel im Schaft kongruent war. Bei einer Patientin kam es aufgrund einer ausgeprägten Antekurvation des Femurschaftes zu einer Perforation der Nagelspitze des geraden kurzen PFN durch die ventrale Femurkortikalis, so dass hier eine zusätzliche Plattenstabilisierung im Bereich der Nagelspitze erforderlich wurde (Abb.5).

An postoperativen lokalen Komplikationen kam es in 12 Fällen zur Ausbildung ausgedehnter Hämatome, von denen 3 revidiert werden mussten, sowie in einem Fall zu einem subkutanen Weich- teilinfekt, der ebenfalls revidiert wurde. Bei dem Patienten mit der $3^{\circ}$ offenen inter- und subtrochantären Defektfraktur, bei der der PFN nach primärer Stabilisierung mit Fixateur externe als Ultima Ratio zum Einsatz kam, trat ein tiefer Infekt auf, der nach Revision über eine Dauerdrainage unter Belassen des Implantates zur Ausheilung gebracht werden konnte.

Gegenüber der früher in unserer Klinik üblichen Versorgung dieser Frakturen mit der $95^{\circ}$-Kondylenplatte konnten die Dauer der Bettruhe bis zum Aufstehen (Mittelwert 3 gegenüber 14 Tage) und der Zeitraum bis zur durchgeführten Vollbelastung (Mittelwert 14 Tage gegenüber 11 Wochen) deutlich gesenkt werden [5].

Der stationäre Aufenthalt betrug durchschnittlich 25,4 Tage; 56\% der Patienten konnten danach wieder direkt in ihre bisherige Umgebung nach Hause oder im Altersheim/Pflegeheim entlassen werden, $14 \%$ wurden in ein anderes Krankenhaus zur Behandlung von Begleitkrankheiten und 30\% zur geriatrischen Rehabilitation verlegt.

Für eine Nachkontrolle nach 4-8 Monaten ( $\varnothing 6$ 6,4 Monate) konnten in einer ersten Serie von 170 Patienten (Implantation 1996-1998) 88 Patienten (52\%) nachuntersucht, 31 (19\%) weitere durch Telefonumfrage erreicht werden. $4 \mathrm{~Pa}$ tienten $(2,3 \%)$ waren während des Krankenhausaufenthaltes verstorben, 25 $(14,7 \%)$ innerhalb von 4 Monaten nach dem Unfallereignis. Sämtliche Todesfälle konnten in keinen kausalen Zusammenhang zur PFN-Implantation gebracht werden.

Bei 86 der nachuntersuchten Patienten (98\%) war die Fraktur zum Untersuchungszeitpunkt knöchern konsolidiert bzw. mit deutlicher Kallusbildung in Konsolidierung begriffen, bei 2 Patienten war die Frakturheilung verzögert und führte in einem Fall zu einer Reosteosynthese mit einem langen PFN, danach kam es auch hier zur knöchernen Ausheilung. Pseudarthrosen traten nicht auf. Implantatversagen (Brüche des Nagels, von Schenkelhalsschrauben oder Verriegelungsbolzen) oder Stressfrakturen am Femurschaft wurden nicht festgestellt. Bei 4 Patienten wurde radiologisch ein „Cutout" der Hüftgleitschraube festgestellt, das bei 3 Patienten zur Revisionsoperation (Hüfttotalendoprothese) führte. Bei weiteren 3 Patienten trat eine Dislokation der Hüftgleitschraube bzw. Schenkelhals- 


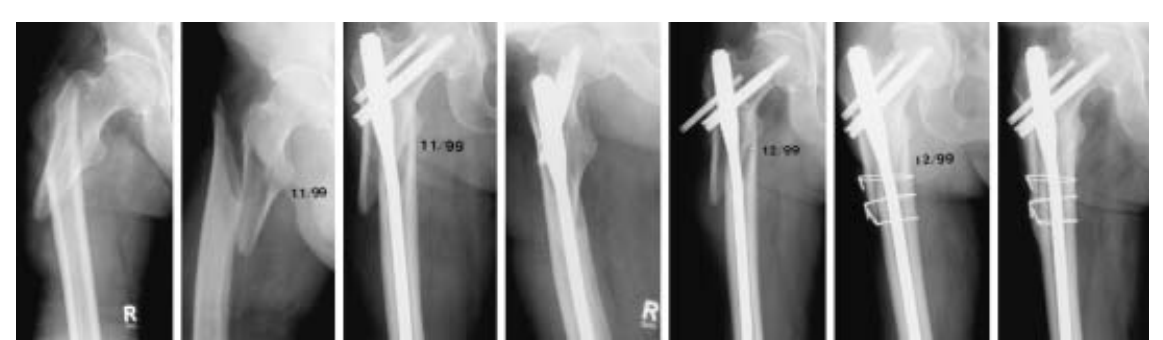

Abb. 6 Komplikation retrograde Dislokation der Hüftgleitschraube.

schraube nach zentral oder lateral auf, hier war ebenfalls eine Revisionsoperation erforderlich (Abb.6).

Bei der Nachuntersuchung belasteten die meisten Patienten (90\%) voll, teilweise mit Unterstützung von Gehhilfen. Gehäufte Schmerzzustände lagen bei $4 \mathrm{~Pa}$ tienten vor (3\%). Die Funktion im Hüftgelenk war bei 73 der 88 nachuntersuchten Patienten mittel- bis endgradig eingeschränkt, vor allem für die Außenrotation, eine Einschränkung im Alltagsleben ergab sich hieraus jedoch nicht.

\section{Ergebnisse der Arbeitsgruppe „Osteosynthesen am proximalen Femur“"}

In einer prospektiv geplanten Multizenterstudie der Arbeitsgruppe „proximales Femur“ der Deutschen Sektion der AO International wurden sämtliche Patienten aufgenommen, welche im Zeitraum vom 1.1.96 bis 31.12.96 in einer der folgenden Kliniken aufgrund einer A2- oder A3-Fraktur operiert wurden [10].

- Berufsgenossenschaftliche Unfallklinik Tübingen

- Unfallchirurgische Abteilung der Chirurgischen Univ.-Klinik Freiburg

- Unfallchirurgische Klinik der HorstSchmitt-Kliniken Wiesbaden

- Abteilung für Unfallchirurgie Chirurg. Univ.-Klinik Heidelberg

- Chirurgische Klinik III, Klinik für Unfall- und Wiederherstellungschirurgie Univ. Leipzig

- Klinikum Lippe Lemgo

- Stadtkrankenhaus Worms

- Unfallchirurgische Abteilung Krankenhaus der Barmherzigen Brüder Regensburg

- Zentralklinikum Augsburg

- Klinik und Poliklinik für Unfallchirurgie, Univ.-Klinikum der Gesamthochschule Essen

- Marienhospital Stuttgart

Es handelte sich um insgesamt 320 Patienten, welche in einer prospektiven Studie nach bestimmten Kriterien ausge- wählt wurden, wobei insbesondere nachstehende Untersuchungskriterien interessierten:
a) Belastbarkeit
b) Früh- und Spätkomplikationen
c) Ausheilungsergebnis

Folgende Osteosyntheseverfahren wurden in den einzelnen Kliniken verwendet. a dynamische Hüftschraube

- dynamische Condylenschraube

- $95^{\circ}$-Condylenplatte

- proximaler Femurnagel

- Gammanagel

54\% des Gesamtkollektivs $=175$ Patienten konnten in einem Nachuntersuchungszeitraum zwischen 6 und 45 Monaten erfasst werden. Die Datenerhebung erfolgte jeweils separat in den einzelnen Kliniken. Die Auswertung wurde von einem Doktoranden der Abteilung Unfallchirurgie der Universität Freiburg (M. Reith) vorgenommen (s. Abb.7-16 u. Tab. 1 u. 2).

\section{Diskussion}

Die Nachuntersuchungen bei Osteosynthesen am proximalen Femur zeigen,

\begin{tabular}{lcc}
\hline Tab. 1 & Intervall zwischen Unfall und Klinikaufnahme & \\
\hline $\begin{array}{l}\text { Anzahl Stunden/Tage von } \\
\text { Unfall bis OP }\end{array}$ & $\begin{array}{l}\text { Anzahl der Patienten } \\
- \text { absolut - }\end{array}$ & $\begin{array}{l}\text { Anzahl der Patienten } \\
- \text { relativ - }\end{array}$ \\
\hline <6 Stunden & 33 & $10,3 \%$ \\
7 - 24 Stunden & 208 & $65,0 \%$ \\
$25-48$ Stunden & 55 & $17,2 \%$ \\
3-10 Tage & 21 & $6,6 \%$ \\
$10-21$ Tage & 0 & $0,0 \%$ \\
21 Tage & 3 & $0,9 \%$
\end{tabular}

Tab. 2 Beurteilung Behandlungsergebnis

\begin{tabular}{llc} 
Ergebnis & Beurteilung durch Patient & Beurteilung durch Arzt \\
\hline ausgezeichnet & 20 & 20 \\
gut & 96 & 113 \\
mäßig & 44 & 32 \\
schlecht & 12 & 6
\end{tabular}

dass es sich um ein Patientenkollektiv mit hohem Alter handelt. Das in der Studie der Arbeitsgemeinschaft für Osteosynthesefragen festgestellte Durchschnittsalter des Patientenkollektivs betrug ca. 83 Jahre, das Verhältnis von Männern zu Frauen lag ca. bei 1:5. Diese Angaben sind auch in der einschlägigen $\mathrm{Li}-$ teratur in gleicher Weise angegeben $[2,4,7]$.

Die Untersuchungen zeigen auch, dass in dieser Altersklasse eine hohe Rate vorbestehender Systemerkrankungen besteht, insbesondere kardiovaskuläre, neurologische und Stoffwechselerkrankungen. Als Frakturursache lag der häusliche Sturz mit über $86 \%$ an erster Stelle, was gleichfalls der zu diesem Thema publizierten Literatur entspricht $[2,4,6,7,9]$.

Das untersuchte Kollektiv wies in knapp $80 \%$ A2-Frakturen und in ca. $20 \%$ A3Frakturen auf. Speziell die Gruppe der A2.2-Frakturen mit einem bereits höheren Instabilitätsgrad war in über der Hälfte der Fälle von A2-Brüchen vertreten. Bei den A3-Frakturen lag die A3.3Fraktur an erster Stelle [10].

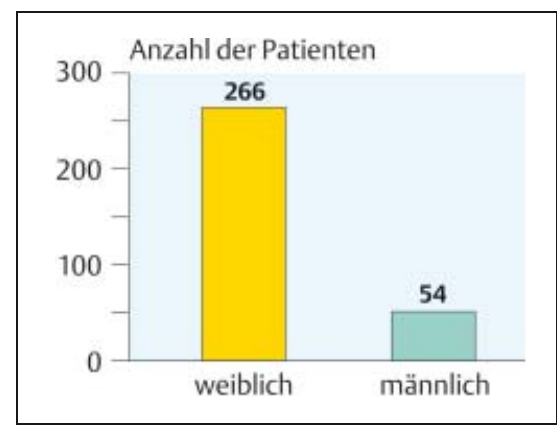

Abb.7 Geschlechtsverteilung der Patienten. 


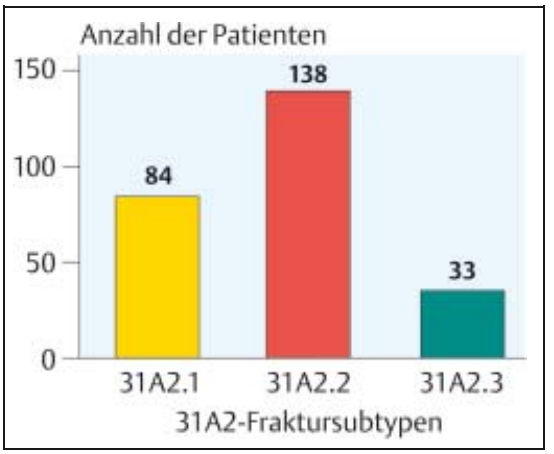

Abb.8 Verteilung der Fraktursubtypen (31A2-Frakturen) - absolute Häufigkeit.

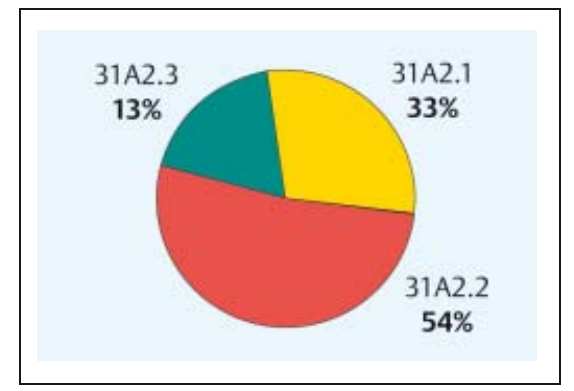

Abb.9 Verteilung der Fraktursubtypen (31A2-Frakturen) - relative Häufigkeit.

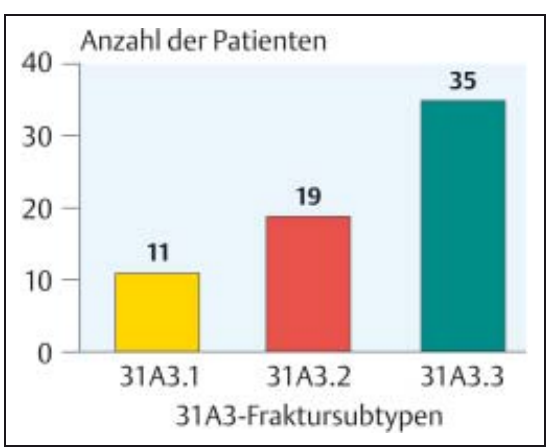

Abb.10 Verteilung der Fraktursubtypen (31A3-Frakturen) - absolute Häufigkeit.

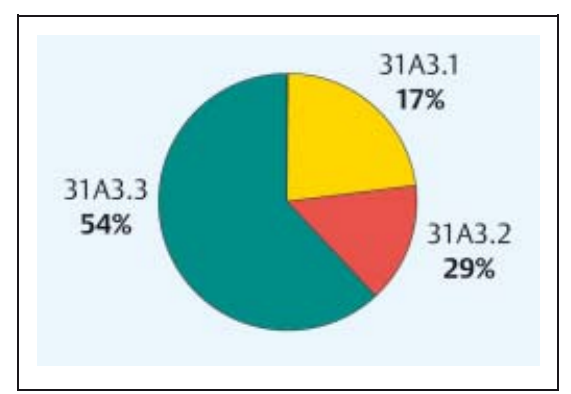

Abb.11 Verteilung der Fraktursubtypen (31A3-Frakturen) - relative Häufigkeit.

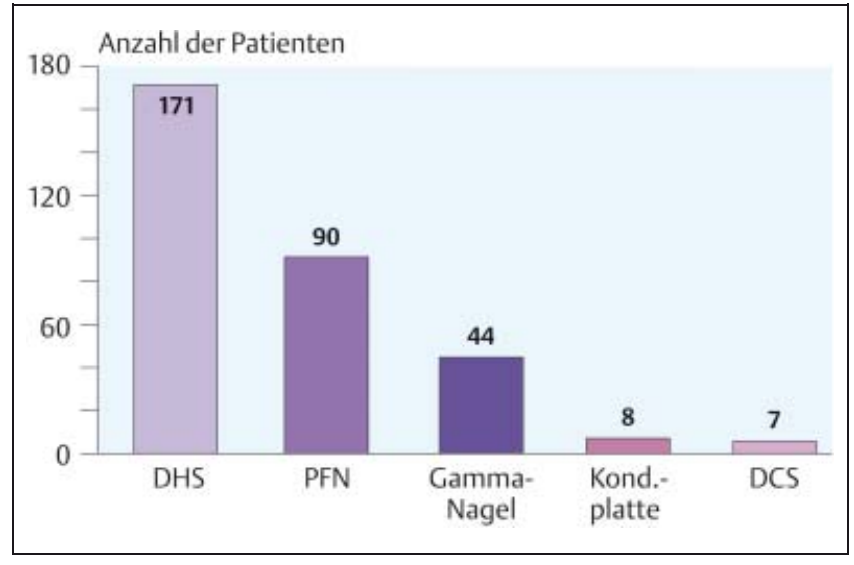

Abb.12 Absolute Häufigkeit der verschiedenen Implantate.

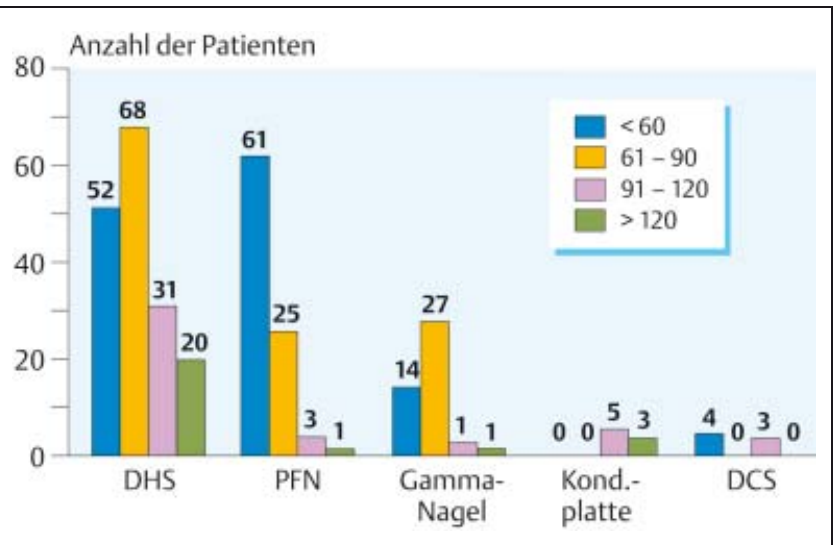

Abb.13 Operationsdauer nach Implantaten.

Abb.14 Gehfähigkeit bei Entlassung nach Implantattyp absolute Häufigkeit.

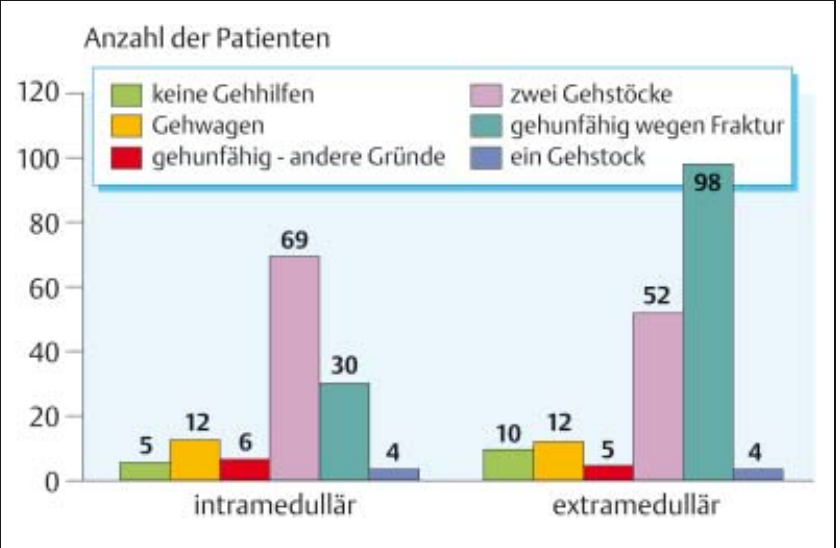

Bei der operativen Versorgung ließ sich nachweisen, dass die intramedullär gelegenen Implantate eine wesentlich frühere mögliche Mobilisierung und damit auch soziale Reintegration erlaubten. Die durchschnittliche Operationsdauer war beispielsweise beim proximalen Femurnagel am kürzesten, wobei in einem Zeitintervall von 60 min mehr als $2 / 3$ der Osteosynthesen mit PFN fertiggestellt werden konnten. Bei der dynamischen Hüftschraube war dies nur in gut 30\% möglich. Was die Belastbarkeit anbetraf, ließ sich wiederum ein Vorteil der intramedullären Kraftträger erkennen, indem mit diesen eine rasche Mobilisierbarkeit unter nach wenigen Tagen erreichter Vollbelastung verwirklicht werden konnte.

Zusammen mit der eigenen prospektiven klinischen Studie mit dem proximalen Femurnagel und nunmehr mehr als 300 behandelten Patienten kann man sagen, dass bei zunehmendem Instabilitätsgrad trochanterer und subtrochanterer Fraktu- 


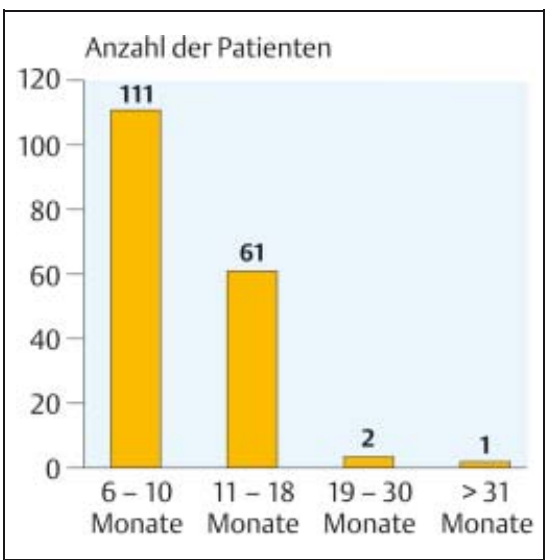

Abb.15 Zeitspanne bis zur Nachkontrolle seit Operationsdatum.

ren mit Vorteil eine intramedulläre Stabilisierungsform gewählt werden sollte. Die anfänglich noch etwas häufiger beobachteten operationstaktischen Fehler z. B. mit dem PFN bzw. Schwierigkeiten bei der Verwendung des Gammanagels sind mittlerweile weitgehend abgestellt und einer durchschrittenen Lernkurve bzw. einer Optimierung der Implantate selbst zu verdanken. Aufgrund der in der Arbeitsgruppe „Osteosynthesen am proximalen Femur" durchgeführten experimentellen Studien an Kunst- und Leichenknochen mit verschiedenen Implantaten und unter Würdigung der klinischen Erfahrungen kann man die Empfehlung aussprechen, dass mit zunehmendem Instabilitätsgrad ein dynamisches intramedulläres Verfahren favorisiert werden sollte. Die dynamische Hüftschraube ist unverändert ein sehr ausgereiftes und gutes Implantat, das eher bei stabilen trochanteren Verletzungen, nicht aber bei den inter- und subtrochanteren Verletzungsformen Verwendung finden sollte, und bei den höhergradigen A2Frakturen nur in Kombination mit einer Trochanterstabilisierungsplatte. Die übrigen Verfahren wie die Winkelplatten und die DCS können in Einzelfällen geeignet sein, sind aber für die Primärversorgung von Frakturen am proximalen Femur eher in den Hintergrund getreten.

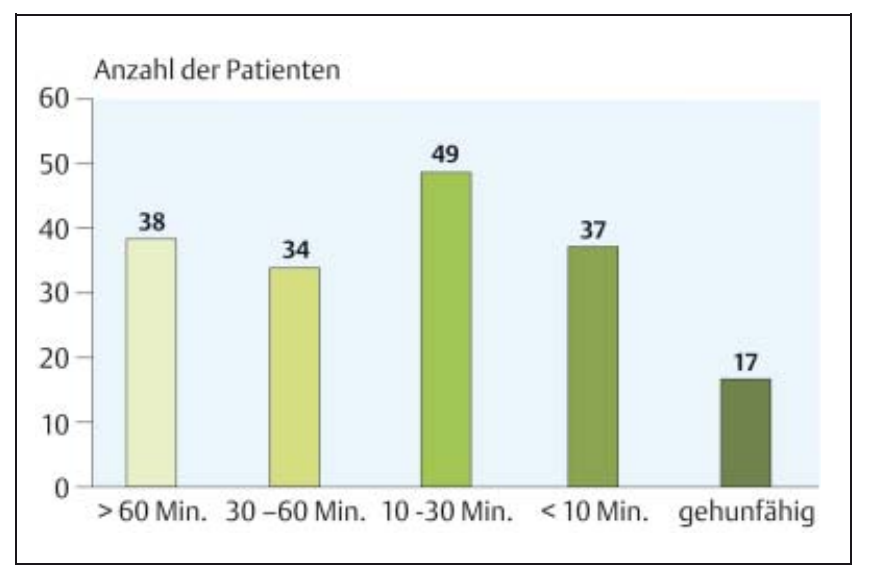

Abb.16 Gehfähigkeit der Nachuntersuchten in Minuten.

\section{Literatur}

${ }^{1}$ Babst R et al. Die DHS-Abstützplatte für die Versorgung der instabilen proximalen Femurfrakturen. Schweiz med Wschr 1993; 123: $566-568$

${ }^{2}$ Bonnaire F, Götschin U, Kuner EH. Früh- und Spätergebnise nach 200 DHS-Osteosynthesen zur Versorgung pertrochanterer Femurfrakturen. Unfallchirurg 1992; 95: 246-253

${ }^{3}$ Götze E, Bonnaire F, Weise K, Friedl HP Osteosynthesen bei instabilen per- und subtrochanteren Femurfrakturen: Experimentelle Untersuchungen mit PFN, Gamma-Nagel, DHS, TSP, 95 Grad-Condylenplatte und UFN/Spiralklinge. Akt Traumatol 1998; 28: 197-204

${ }^{4}$ Hoffmann $\mathrm{R}$ et al. Classic-Nail vs. dynamische Hüftschraube (DHS). Unfallchirurg 1999; 102: $182-190$

${ }^{5}$ Kaiser SP. Die Behandlung per- und subtrochanterer Frakturen mit der Kondylenplatte. Dissertation Med Fakultät der EberhardKarls-Universität Tübingen 1997

${ }^{6}$ Obertacke U, Nast-Kolb D. Besonderheiten des Unfalls, der Verletzung und der chirurgischen Versorgung im höheren Lebensalter. Unfallchirurg 2000; 103: 227-239

${ }^{7}$ Schwab E, Höntzsch D, Weise K. Die Versorgung instabiler per- und subtrochantärer Fe- murfrakturen mit dem Proximalen Femurnagel (PFN). Akt Traumatol 1998; 28 (2): $56-60$

${ }^{8}$ Stürmer KM et al. Wandel bei der Osteosynthese pertrochantärer und subtrochantärer Femurfrakturen. H.z.d. Unfallchir, H 232 K.E.Rehm (Hrsg.) Springer Berlin Heidelberg 1993; 99-121

${ }^{9}$ Wagner S, Rüter A. Per- und subtrochantäre Femurfrakturen. Unfallchirurg 1999; 102: 206-222

${ }^{10}$ Weise K et al. Abschlussbericht Arbeitsgruppe „Osteosynthesen am proximalen Femur“ der Deutschen Sektion der Arbeitsgemeinschaft für Osteosynthesefragen DAO-aktuell 2000; 2000

Prof. Dr. med. K. Weise

Ärztlicher Direktor

Dr. med. E. Schwab

Oberarzt

BG-Unfallklinik

Schnarrenbergstr. 95

72076 Tübingen 